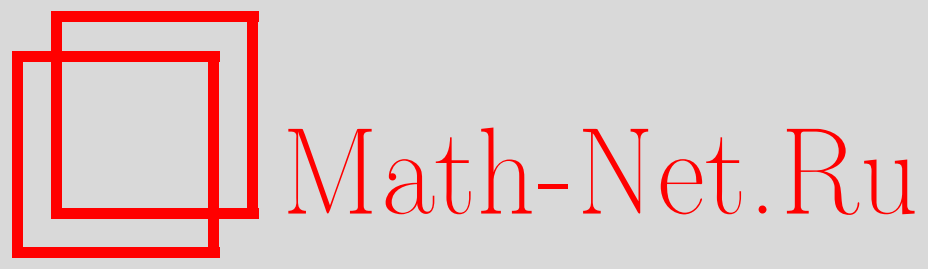

В. С. Рабинович, С. Г. Самко, Сингулярные интегральные операторы в весовых пространствах Лебега с переменными показателями на сложных карлесоновских кривых, Функи. анализ и его прил., 2012, том 46, выпуск 1, 87-92

DOI: https://doi.org/10.4213/faa3061

Использование Общероссийского математического портала MathNet.Ru подразумевает, что вы прочитали и согласны с пользовательским соглашением

http://www . mathnet.ru/rus/agreement

Параметры загрузки:

IP : 3.85 .5 .30

26 апреля 2023 г., 16:32:54

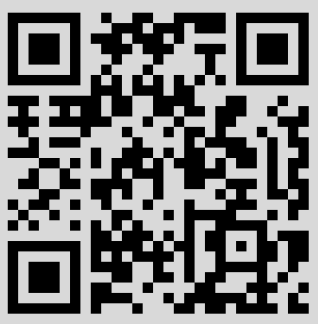


Предложение 13. Пусть $\varphi$ есть l-непрерывное интервальное округление и $(A, B, \alpha, \beta)$ - его функииональная модель. Для того чтобы $\varphi$ было идемпотентно аддитивным, необходимо и достаточно, чтобы функиии $\alpha$ и $\beta$ были убывающими.

Условие непрерывности позволяет значительно упростить функциональную модель.

Следствие 14. Непрерывное интервальное округление $\varphi$ можно задавать условием

$$
E_{\varphi}=\{(x, y): y \geqslant \gamma(x), x \leqslant \delta(y)\},
$$

выбирая произвольные числа $a, b$ и непрерывные невозрастающие функиии $\gamma, \delta$ на $(-\infty, a])$ u $[b,+\infty)$, удовлетворяющие условиям

(a) $\delta(\gamma(x)) \geqslant x, \gamma(\delta(y)) \leqslant y$;

(b) $\lim _{x \rightarrow-\infty} \gamma(x)=+\infty, \lim _{y \rightarrow+\infty} \delta(y)=-\infty$.

Автор выражает искреннюю признательность Т. Э. Каминскому за постановку задач и полезные обсуждения.

\title{
ЛитеРАТУРА
}

[1] Г. Биркгоф, Теория структур, ИЛ, М., 1952. [2] Т. E. Kaminsky, V. Kreinovich, Notes on intuitionistic fuzzy sets, 4:3 (1998), 57-64. [3] Т. Э. Каминский, в кн.: Исследования по математическому анализу и методике преподавания математики, Русь, Вологда, 2000, 23-36. [4] U. Kulisch, Numer. Math., 18:1 (1971), 1-17. [5] G. L. Litvinov, The Maslov dequantization, idempotent and tropical mathematics: A brief introduction, http://arxiv.org/abs/math/0507014v1. [6] Е. В. Шульман, J. London Math. Soc. (2), 54:1 (1996), 111-120.

Вологодский государственный педагогический университет e-mail: kryukovanastya@yahoo.com

Поступило в редакцию 18 января 2010 г.

\section{Сингулярные интегральные операторы в весовых пространствах Лебега с переменными показателями на сложных карлесоновских кривых}

\author{
(c) 2012. В. С. РАБинович, С. Г. Самко
}

Памяти профессора И. Б. Симоненко посвящается

1. Введение. В работе [15] И. Б. Симоненко впервые ввел локальный метод исследования фредгольмовости одномерных и многомерных сингулярных интегральных операторов (СИО). Посредством этого метода исследование фредгольмовости СИО $A=a I+b S_{\Gamma}$ с непрерывными коэффициентами $a$ и $b$, действующих в $L^{p}(\Gamma), 1<p<\infty$, на кривых Ляпунова $Г$, сводилось к исследованию локальных представителей оператора $A$ в каждой точке $t_{0} \in \Gamma$, являющихся простейшими операторами типа $A_{t_{0}}=a\left(t_{0}\right) I+b\left(t_{0}\right) S_{\mathbb{R}}$, действуюшими в $L^{p}(\mathbb{R})$. Этот метод был применен в [14] к СИО с кусочно непрерывными коэффициентами, действующими в пространствах $L^{p}(\Gamma, w)$ со степенным весом 
$w$ на кривых Ляпунова Г. Локальными представителями в этом случае являются однородные операторы $A=a I+b S_{\mathbb{R}}$, действующие в $L^{p}(\mathbb{R})$, где $a, b-$ кусочно постоянные функции только с одним разрывом в точке 0 , и условие обратимости таких операторов дается в терминах преобразований Меллина их ядер.

В работе применяется локальный принцип Симоненко для исследования фредгольмовости сингулярных интегральных операторов, действующих в весовых пространствах Лебега с переменными показателями на некоторых сложных карлесоновских кривых с коэффициентами, допускающими осциллирующие разрывы. Локальными представителями в этом случае являются nсевдодифберенииальные операторы Меллина с переменными символами. Символ локального представителя в сингулярной точке $t$ кривой, веса и коэффициентов содержит все их локальные характеристики: вращения и осцилляции кривой, осцилляции весов, коэффициентов и значение $p(t)$ показателя пространства Лебега в точке $t$. Выражение для символа объясняет появление двойных логарифмических спиралей и логарифмических лунок в локальных спектрах сингулярных интегральных операторов. Отметим, что в теории Гохберга, Крупника и Спитковского ([4], [16]) для СИО, действующих в пространствах $L^{p}$ с весами Макенхаупта на кривых Ляпунова, типичными локальными спектрами являются циркулярные кривые и циркулярные лунки.

В заметке результаты статей [2], [3], [12], где рассматривались постоянные показатели $p$, распространяются на пространства с переменными показателями Лебега. Это распространение существенно нетривиально, так как, в частности, пространства $L^{p(\cdot)}(\mathbb{R}), L^{p(\cdot)}\left(\mathbb{R}_{+}, d r / r\right)$ не инвариантны соответственно относительно сдвигов и подобий, что является очень важным для методов указанных статей.

Отметим, что теория СИО с кусочно непрерывными коэффициентами, действующих в пространствах $L^{p}(\Gamma, w)$, где $\Gamma$ - общая кривая Карлесона, а $w-$ вес Макенхаупта, развита в монографии [1] посредством факторизации ВинераХопфа и теории субмультипликативных функций (см. также ссылки, приведенные в этой монографии.) В [5]-[7] некоторые результаты из [1] распространены на алгебры СИО, действующих в пространствах Лебега с переменными показателями.

Надо отметить большой интерес, появившийся недавно, к исследованию классических операторов анализа: СИО, операторов Харди, псевдодифференциальных операторов в весовых пространствах Лебега с переменными показателями. Мы упомянем только работы [8]-[11], [13]. См. также ссылки, приведенные там.

2. Кривые, коэффициенты, сингулярные интегралы. Множество $\gamma \subset \mathbb{C}$ называется простой гладкой кривой, если существует гомоморфизм $\varphi:[0,1] \rightarrow \gamma$, такой, что $\varphi \in C^{\infty}(0,1)$ и $\varphi^{\prime}(r) \neq 0$ для всех $r \in(0,1)$. Точки $\varphi(0)$ и $\varphi(1)$ называются концевыми точками кривой $\gamma$. Мы называем множество $\Gamma \subset \mathbb{C}$ сложной кривой, если $\Gamma=\bigcup_{k=1}^{K} \Gamma_{k}$, где $\Gamma_{1}, \ldots, \Gamma_{K}-$ ориентированные и спрямляемые простые кривые, попарно не пересекающиеся, за исключением, возможно, концевых точек. Узлом кривой Г называется точка, которая является концом по крайней мере одной из дуг $\Gamma_{1}, \ldots, \Gamma_{K}$. Множество узлов обозначается через $\mathscr{F}$.

Будем говорить, что функция $f:(0, \varepsilon) \rightarrow \mathbb{C}$, принадлежащая $C^{\infty}$, является медленно осииллирующей в нуле, если $\sup _{r \in(0, \varepsilon)}\left|(r d / d r)^{k} f(r)\right|<\infty$ для 
$k=0,1,2, \ldots$ и $\lim _{r \rightarrow 0} r f^{\prime}(r)=0$. Примером функции, которая медленно осциллирует в нуле, может служить функция $f(r)=g(\log (-\log r))$, где $g$ принадлежит пространству $C_{b}^{\infty}(\mathbb{R})$ всех бесконечно дифференцируемых функций на $\mathbb{R}$, ограниченных вместе со всеми производными.

Пусть $\Gamma$ - сложная кривая и $t_{0} \in \mathscr{F}$. Будем говорить, что $\Gamma$ медленно осииллирует в точке $t_{0}$, если существует $\varepsilon>0$, такое, что часть $\Gamma\left(t_{0}, \varepsilon\right)=\{t \in$ $\left.\Gamma:\left|t_{0}-t\right|<\varepsilon\right\}=\left\{t_{0}\right\} \cup \Gamma_{t_{0} 1} \cup \cdots \cup \Gamma_{t_{0} n_{t_{0}}}$ кривой $\Gamma$ такова, что

$$
\Gamma_{t_{0} j}=\left\{z \in \mathbb{C}: z=t_{0}+r e^{i \varphi_{t_{0}, j}(r)}, r \in(0, \varepsilon), j=1, \ldots, n_{t_{0}}\right\},
$$

где $\varphi_{t_{0}, j}(r)=\theta_{t_{0}}(r)+\theta_{t_{0}}(r), \theta_{t_{0}}, \theta_{t_{0}}, \ldots, \theta_{t_{0} n_{t_{0}}}-$ вещественнозначные $C^{\infty}$-функции, такие, что

$0 \leqslant m_{1}<\theta_{t_{0} 1}(r)<M_{1}<m_{2}<\theta_{t_{0} 2}(r)<M_{2}<\cdots<m_{n_{t_{0}}}<\theta_{t_{0} n_{t_{0}}}(r)<M_{n_{t_{0}}}<2 \pi$ для всех $r \in(0, \varepsilon)$ с некоторыми $m_{j}, M_{j}$ (вообще говоря, зависящими от $t_{0}$ ). Мы предполагаем, что функции $r \theta_{t_{0}}^{\prime}(r), r \theta_{t_{0} 1}^{\prime}(r), \ldots, r \theta_{t_{0} n_{t_{0}}}^{\prime}(r)$ медленно осциллируют в нуле.

Например, если $\theta_{t_{0}}(r)+\theta_{t_{0} j}(r)=\delta_{t_{0}} \log r+\mu_{t_{0} j}\left(j=1, \ldots, n_{t_{0}}\right)$ и $0 \leqslant \mu_{t_{0} 1}<$ $\mu_{t_{0} 2}<\cdots<\mu_{t_{0} n_{t_{0}}}<2 \pi$, то приведенные выше предположения выполняются.

Сложную кривую, медленно осциллирующую в каждом узле, будем называть медленно осииллирующей сложной кривой. Класс медленно осциллирующих сложных кривых в дальнейшем обозначается через $\mathscr{C}_{S O}$. Принимая во внимание, что $\left|d\left(r e^{i \varphi(r)}\right)\right|=\sqrt{\left(1+\left(r \varphi^{\prime}(r)\right)^{2}\right.} d r$, нетрудно увидеть, что медленно осциллирующая сложная кривая является карлесоновской кривой (см. [1, с. 2]).

Пусть $p: \Gamma \rightarrow(1, \infty)$ - измеримая функция, $1<p_{-} \leqslant p(t) \leqslant p_{+}<\infty, t \in \Gamma$, и $|p(t)-p(\tau)| \leqslant A / \log \frac{1}{|t-\tau|}$ для любых точек $t, \tau \in \Gamma \backslash \mathscr{F}$, таких, что $|t-\tau|<1 / 2$. Если $t_{0} \in \mathscr{F}$, то мы будем предполагать, что существует $\varepsilon>0$, такое, что функция $p_{t_{0} j}(r)=p\left(t_{0}+r e^{i \varphi_{t_{0}, j}(r)}\right)=p_{t_{0}}(r), r \in(0, \varepsilon)$, не зависит от $j$ и $p_{t_{0}}(r)$ удовлетворяет условиям $\left|p_{t_{0}}(r)-p_{t_{0}}(\rho)\right| \leqslant A / \log \frac{1}{|\log (r / \rho)|}$ для $1 / \sqrt{e} \leqslant r / \rho \leqslant \sqrt{e}$, и $\left|p_{t_{0}}(r)-p_{t_{0}}(0)\right| \leqslant C / \log (2+|\log r|), r, \rho \in(0, \varepsilon)$. Из этих условий следует, что $p_{t_{0}}(\cdot)$ - непрерывная функция в нуле и $\lim _{r \rightarrow+0} p_{t_{0}}(r)=p_{t_{0}}(0)=p\left(t_{0}\right)$. Будем обозначать через $\mathscr{P}(\Gamma)$ класс переменных показателей, удовлетворяющих приведенным выше условиям.

Пусть $w: \Gamma \rightarrow[0, \infty]$ - измеримая функция, называемая в дальнейшем весом. Весовое пространство Лебега $L^{p(\cdot)}(\Gamma, w)$ с переменным показателем $p(\cdot)$ определяется посредством модуляра $I^{p}(w, f):=\int_{\Gamma}|w(\tau) f(\tau)|^{p(\tau)}|d \tau|<\infty$ и нормы $\|f\|_{L^{p(\cdot)(\Gamma, w)}}=\inf \left\{\lambda>0: I^{p}(w, f / \lambda) \leqslant 1\right\}$. Мы рассматриваем веса на $\mathbb{R}_{+}$, имеющие форму $w(r)=\exp v(r)$, где функция $\varkappa_{w}(r)=r v^{\prime}(r)$ медленно осциллирует, и обозначаем класс таких весов через $\mathscr{R}$. Положим $\varkappa_{w}^{+}=\limsup _{r \rightarrow 0} \varkappa_{w}(r)$, $\varkappa_{w}^{-}=\liminf _{r \rightarrow 0} \varkappa_{w}(r)$. Пусть $w: \Gamma \rightarrow[0,+\infty]-$ вес на кривой Г. Мы предполагаем, что для каждой точки $t_{k} \in \mathscr{F}$ существует окрестность $U_{k}$, такая, что $w$ и $w^{-1}$ принадлежат $L^{\infty}\left(\Gamma \backslash \bigcup_{t_{k} \in \mathscr{F}}\left(\Gamma \cap U_{k}\right)\right)$. Мы говорим, что вес $w$ медленно осциллирует, если для каждой точки $t_{0} \in \mathscr{F}$ и для каждого $j \in\left\{1, \ldots, n_{t_{0}}\right\}$ функция $w_{t_{0}}(r)=w\left(t_{0}+r e^{i \varphi_{t_{0}, j}(r)}\right)=e^{v_{t_{0}}(r)}, r \in(0, \varepsilon)$, не зависит от $j$ и $w_{t_{0}}(r)$ есть вес, принадлежащий $\mathscr{R}$. Обозначим через $\mathscr{A}_{p(\cdot)}(\Gamma)$ класс медленно осциллирующих весов, таких, что $-1 / p\left(t_{k}\right)<\varkappa_{w_{t_{k}}}^{-} \leqslant \varkappa_{w_{t_{k}}}^{+}<1-1 / p\left(t_{k}\right)$ для любой точки $t_{k} \in \mathscr{F}$. 
Функция $a: \Gamma \rightarrow \mathbb{C}$ называется медленно осииллирующей на $\Gamma, a \in P S O(\Gamma)$, если $a \in C^{\infty}(\Gamma \backslash \mathscr{F})$ и для каждого узла $t_{0} \in \mathscr{F}$ функция $a\left(t_{0}+r e^{i \varphi_{t_{0}, j}(r)}\right)=$ $a_{t_{0}, j}(r), r \in(0, \varepsilon), j \in\left\{1, \ldots, n_{t_{0}}\right\}$, медленно осциллирует в точке $r=0$.

Сингулярный интегральный оператор Коши на кривой Г определяется как

$$
\left(S_{\Gamma} f\right)(t)=\lim _{\varepsilon \rightarrow 0} \int_{\Gamma \backslash \Gamma\left(t_{0}, \varepsilon\right)} \frac{f(\tau) d \tau}{\tau-t}, \quad t \in \Gamma .
$$

Теорема 1. Пусть $\Gamma \in \mathscr{C}_{S O}, w \in \mathscr{A}_{p(\cdot)}(\Gamma), p \in \mathscr{P}(\Gamma)$. Тогда оператор $S_{\Gamma}: L^{p(\cdot)}(\Gamma, w) \rightarrow L^{p(\cdot)}(\Gamma, w)$ ограничен.

3. Фредгольмовость. Можно доказать, что оператор $S_{\Gamma}: L^{p(\cdot)}(\Gamma, w) \rightarrow$ $L^{p(\cdot)}(\Gamma, w)$ является оператором локального типа в смысле Симоненко. Это означает, что для любых функций $\varphi, \psi \in C^{\infty}(\Gamma)$, таких, что $\operatorname{supp} \varphi \cap \operatorname{supp} \psi=\varnothing$, оператор $\varphi S_{\Gamma} \psi I: L^{p(\cdot)}(\Gamma, w) \rightarrow L^{p(\cdot)}(\Gamma, w)$ компактен. Будем говорить, что $A: L^{p(\cdot)}(\Gamma, w) \rightarrow L^{p(\cdot)}(\Gamma, w)$ локально обратим в точке $t_{0} \in \Gamma$, если существует окрестность $U_{t_{0}}$ точки $t_{0}$ и ограниченные операторы $\mathscr{L}_{t_{0}}, \mathscr{R}_{t_{0}}$, такие, что $\mathscr{L}_{t_{0}} A \chi_{U_{t_{0}}} I=\chi_{U_{t_{0}}} I$ и $\chi_{U_{t_{0}}} A \mathscr{R}_{t_{0}}=\chi_{U_{t_{0}}} I$.

Предложение (локальный принцип Симоненко ([15], [14])). Оператор локального типа $A: L^{p(\cdot)}(\Gamma, w) \rightarrow L^{p(\cdot)}(\Gamma, w)$ является фредгольмовым оператором тогда и только тогда, когда он локально обратим в каждой точке $t \in \Gamma$.

Замечание 1. Отметим, что локальный принцип Симоненко был доказан в [15] для операторов, действующих в пространствах $L^{p}$ с постоянным показателем. Однако он остается справедливым также для операторов, действующих в $L^{p(\cdot)}(\Gamma, w)$.

Введем обозначения, необходимые для формулировки основного результата заметки.

(i) Пусть $t_{0} \in \mathscr{F}$. Положим $\varepsilon_{k}=1$, если $t_{0}$ - начальная точка простой ориентированной кривой $\Gamma_{t_{0} k}$, и пусть $\varepsilon_{k}=-1$, если $t_{0}-$ конечная точка этой кривой. Определим отображение $\nu:[0,2 \pi) \times(\mathbb{C} \backslash i \mathbb{Z}) \rightarrow \mathbb{C}$ формулой

$$
\nu(\delta, z)= \begin{cases}\operatorname{cth}(\pi z), & \delta=0, \\ e^{(\pi-\delta) z / \operatorname{sh}(\pi z),} & \delta \in(0,2 \pi),\end{cases}
$$

и пусть

$$
s_{j k}^{t_{0}}(r, z)= \begin{cases}\varepsilon_{k} \nu\left(2 \pi+\theta_{t_{0} j}(r)-\theta_{t_{0} k}(r), z\right), & j<k, \\ \varepsilon_{k} \nu(0, z), & j=k, \\ \varepsilon_{k} \nu\left(\theta_{t_{0} j}(r)-\theta_{t_{0} k}(r), z\right), & j>k .\end{cases}
$$

Положим

$\hat{\sigma}_{t_{0}}\left(S_{\Gamma}\right)(r, z)=\left(s_{j k}^{t_{0}}\left(r, \frac{z+i\left(1 / p\left(t_{0}\right)+\varkappa_{w}(r)\right)}{1+i r \theta_{t_{0}}^{\prime}(r)}\right)\right)_{j, k=1}^{n_{t_{0}}}, \quad(r, z) \in \mathbb{R}_{+} \times \mathbb{R}$.

(ii) Если $a \in P S O(\Gamma)$, то $\hat{\sigma}_{t_{0}}(a I)=\operatorname{diag}\left(a_{t_{0}, 1}(r), \ldots, a_{t_{0}, n_{t_{0}}}(r)\right)$.

(iii) Пусть $t_{0} \in \Gamma \backslash \mathscr{F}$. Тогда $\hat{\sigma}_{t_{0}}\left(S_{\Gamma}\right)=\operatorname{sign} \xi, \xi \in \mathbb{R}, \hat{\sigma}_{t_{0}}(a I)=a\left(t_{0}\right)$.

(iv) Если $A=a I+b S_{\Gamma}$, то $\hat{\sigma}\left(A^{t_{0}}\right)=\hat{\sigma}_{t_{0}}(a I)+\hat{\sigma}_{t_{0}}(b I) \hat{\sigma}_{t_{0}}\left(S_{\Gamma}\right)$. 
Теорема 2. Пусть $A=a I+b S_{\Gamma}, a, b \in P S O(\Gamma), \Gamma \in \mathscr{C}_{S O}, w \in \mathscr{A}_{p(\cdot)}(\Gamma)$, $p(\cdot) \in \mathscr{P}(\Gamma)$. Тогда $A: L^{p(\cdot)}(\Gamma, w) \rightarrow L^{p(\cdot)}(\Gamma, w)$ является фредгольмовим оператором в том и только в том случае, когда

$$
\liminf _{r \rightarrow \infty} \inf _{\lambda \in \mathbb{R}}\left|\operatorname{det} \hat{\sigma}\left(A^{t}\right)(r, \lambda)\right|>0
$$

для каждой точки $t \in \mathscr{F} u$

$$
a^{2}(t)-b^{2}(t) \neq 0
$$

для каждой точки $t \in \Gamma \backslash \mathscr{F}$.

Фредгольмов индекс оператора А определяется формулой

$$
\operatorname{index} A=-\sum_{j=1}^{K} \frac{1}{2 \pi}\left[\arg \frac{a(t)+b(t)}{a(t)-b(t)}\right]_{t \in \Gamma_{j}}-\sum_{j=1}^{L} \frac{1}{2 \pi} \lim _{r \rightarrow 0}\left[\arg \operatorname{det} \sigma\left(A^{t_{j}}\right)(r, z)\right]_{z=-\infty}^{\infty},
$$

где $K$ есть число всех ориентированных простых кривых, формирующих сложную кривую $\Gamma$, а $L-$ число всех ее узлов.

Доказательство теоремы 2 основывается на методах, развитых в статьях [2], [3], [12]. Эти методы сводят вопрос о локальной обратимости локальных представителей СИО в сингулярных точках кривой Г к вопросу локальной обратимости псевдодифференциальных операторов Меллина с переменными символами. Применяя методы и результаты из [13], касающиеся ограниченности и фредгольмовости псевдодифференциальных операторов, действующих в весовых пространствах $L^{p(\cdot)}\left(\mathbb{R}^{n}, w\right)$ с переменными показателями $p(\cdot)$, мы доказываем теорему 2.

Замечание 2. Условие (4) содержит все локальные характеристики СИО $A$ в сингулярной точке $t$ : характеристику вращения $r \theta_{t}^{\prime}(r)$ кривой $\Gamma$ в точке $t \in \mathscr{F}$, характеристики $\theta_{t j}(r)$ осцилляций кривых $\Gamma_{t j}$, формирующих узел $t \in \mathscr{F}$, характеристику $\varkappa_{w}(r)$ веса в точке $t \in \mathscr{F}$ и значение $p(t)$ переменного показателя в точке $t \in \mathscr{F}$. Если $t \in \Gamma \backslash \mathscr{F}$, то условие $(5)$ не зависит от $p(\cdot)$ и совпадает с хорошо известным условием локальной обратимости СИО, действующих в пространствах $L^{p}(\Gamma), 1<p<\infty$, на кривых Ляпунова $Г$ и имеющих непрерывные коэффициенты.

Замечание 3. Результаты этой заметки переносятся на операторы $A_{N M}=$ $\sum_{j=1}^{N} \prod_{k=1}^{M} A_{j k}$, где $A_{j k}=a_{j k} I+b_{j k} S_{\Gamma}$ и $a_{j k}, b_{j k} \in P S O(\Gamma)$, а также на операторы из банаховой алгебры, порожденной операторами $A_{N M}$, действующими в $L^{p(\cdot)}(\Gamma, w)$.

\section{ЛитеРАТУРА}

[1] A. Böttcher, Yu. I. Karlovich, Carleson Curves, Muckenhoupt Weights, and Toeplitz Operators, Progress in Math., vol. 154, Birkhauser, Basel, 1997. [2] A. Böttcher, Yu. I. Karlovich, V. S. Rabinovich, J. Operator Theory, 43:1 (2000), 171-198. [3] A. Böttcher, Yu. I. Karlovich, V. S. Rabinovich, Manuscripta Math., 95:3 (1998), 363376. [4] I. C. Gohberg, N. Ya. Krupnik, I. M. Spitkovsky, Integral Equations Operator Theory, 17:3 (1993), 322-327. [5] A. Yu. Karlovich, in: Operator Algebras, Operator Theory and Applications, Operator Theory Adv. Appl., vol. 195, Birkhäuser, Basel, 2010, 185-212. [6] A. Yu. Karlovich, in: Topics in Operator Theory 1: Operators, Matrices 
and Analytic Functions, Operator Theory Adv. Appl., vol. 202, 2010, 321-336. [7] A. Yu. Karlovich, http://arxiv.org/abs/1002.4813v1. [8] V. Kokilashvili, S. Samko, Proc. A. Razmadze Math. Inst., 131 (2003), 61-78. [9] V. Kokilashvili, S. Samko, Proc. A. Razmadze Math. Inst., 138 (2005), 106-110. [10] V. Kokilashvili, V. Paatashvili, S. Samko, in: Operator Theory Adv. Appl., vol. 170 (dedicated to 70th birthday of Prof. I. B. Simonenko), Birkhäuser, Basel, 2007, 167-186. [11] V. Kokilashvili, N. Samko, S. Samko, Math. Nachr., 280:9-10 (2007), 1145-1156. [12] В. С. Рабинович, Изв. РАН, сер. матем., 60:6 (1996), 169-200. [13] V. Rabinovich, S. Samko, Integral Equations and Operator Theory, 60:4 (2008), 507-537. [14] И. Б. Симоненко, Чинь Нгок Минь, Локалъный метод в теории одномерных сингулярных интегралъных уравнений с кусочно-непрерывными коэффиииентами. Нетеровость, Изд-во Ростовского ун-та, 1986. [15] И. Б. Симоненко, Изв. АН СССР, сер. матем., 29:3 (1965), 567-586; 29:4 (1965), 757-782. [16] I. M. Spitkovsky, J. Funct. Anal., 105:1 (1992), 129-143.

Национальный политехнический институт Мексики, e-mail: vladimir.rabinovich@gmail.com

Поступило в редакцию Университет Алгарве, Португалия e-mail: ssamko@ualg.pt

\title{
Об эйлеровых характеристиках многообразий особенностей волновых фронтов*
}

\author{
(c) 2012. В. Д. СЕдых
}

Все понятия и факты теории особенностей лежандровых отображений [1], которые мы здесь используем, кратко изложены в нашей предыдущей заметке [5].

Пусть $B$ - гладкое (класса $C^{\infty}$ ) многообразие размерности $n, E$ - гладкое $(2 n-1)$-мерное многообразие, снабженное контактной структурой, и $\rho: E \rightarrow B$ - лежандрово расслоение. Рассмотрим гладкое компактное (без края) лежандрово подмногообразие $L$ в $E$ и лежандрово отображение $f=\rho \circ i: L \rightarrow B$, где $i: L \rightarrow E-$ тождественное вложение. Образ $\mathscr{F}=f(L)$ отображения $f$ называется (волновым) фронтом. Мы будем говорить, что утверждение, касающееся лежандровых отображений или их фронтов, справедливо для отображения $f$ или фронта $\mathscr{F}$ общего положения, если оно имеет место для вложений $i$ из некоторого открытого всюду плотного подмножества в пространстве всех вложений $i: L \rightarrow E$ (в $C^{\infty}$-топологии).

При $n \leqslant 6$ особенности отображения $f$ общего положения лежандрово эквивалентны особенностям в нуле отображения

$$
\mathbb{R}^{n-1} \rightarrow \mathbb{R}^{n}, \quad(\bar{t}, \bar{q}) \mapsto\left(-S(\bar{t}, \bar{q})+\bar{t} \frac{\partial}{\partial \bar{t}} S(\bar{t}, \bar{q}),-\frac{\partial}{\partial \bar{t}} S(\bar{t}, \bar{q}), \bar{q}\right),
$$

где $\bar{t}=\left(t_{1}, \ldots, t_{k}\right), \bar{q}=\left(q_{k+1}, \ldots, q_{n-1}\right)$, а $S=S(\bar{t}, \bar{q})-$ гладкая функция одного из следующих типов ( $\mu$ целое): 